\title{
Die konspirationistische Internationale im Covid-Kontext: Verschwörungserzählungen, Apokalypsen und Affektkulturen einer „neuen Rechten“ in Lateinamerika
}

Patrick Eser

Die Bilder von den Protesten, die sich in verschiedenen Ländern Lateinamerikas gegen die Maßnahmen zur Eindämmung des Corona-Virus richteten, ähneln denen homologer Demonstrationen in Europa seit dem Sommer 2020. Zwei Merkmale stechen hervor: die heterogene soziale $\mathrm{Zu}$ sammensetzung und „Buntheit“ der Akteur"innen der Proteste sowie die Wiederkehr gleichnamiger Forderungen, Slogans und Symbole. Es ist die Rede von einer unterdrückerischen "Neuen Weltordnung“, die eigenen, bedrohten Freiheitsrechte werden vehement bekräftigt, die Verwendung der Atemschutzmaske wird ebenso radikal abgelehnt wie die Impfung. Wiederholt ist das Symbol „5G“ zu sehen, die neue Generation der Mobilfunktechnologie wird offenbar ebenfalls als Bedrohung wahrgenommen. Das Symptom der globalen Ausbreitung dieser Art von Protesten wirft Fragen auf, deren Klärung einen schärferen Blick auf gegenwärtige Protestkulturen im Kontext der Globalisierung ermöglichen soll. Auch wäre zu erörtern, inwiefern sich darin die Etablierung einer rechten Spielart von Globalisierungskritik abzeichnet, die erfolgreich an Formen, Symbole und Rhetorik der mittlerweile erschöpften und wirkungslos gebliebenen linken Spielart anschließen kann. Ein kursorischer Blick auf Äußerungen der Protestkulturen in Lateinamerika soll eine erste Annäherung an dieses politische wie gegenwartskulturelle Phänomen ermöglichen, zu dessen festem Repertoire Verschwörungsnarrative gehören. Da der Kontext der Corona-Pandemie eine dynamisierende Wirkung auf politische UnmutsäuBerungen hat, weisen die folgenden Ausführungen Züge einer Momentaufnahme auf. Der Schwerpunkt wird im Folgenden auf die Frage gelegt, ob und inwiefern sich im Umfeld dieser Phänomene eine neue Spielart der politischen Rechten in Lateinamerika ausmachen lässt. 


\section{Zeitdiagnostische Perspektiven auf ein Phänomen der Gegenwart}

An zeitdiagnostischen Aufklärungsversuchen, zunächst journalistischer, zunehmend auch akademischer Provenienz, hinsichtlich der Proteste gegen die staatlichen Maßnahmen zur Eindämmung der Pandemie mangelt es nicht. Sie untersuchen die Protestkulturen im nationalen Kontext, wo diese an Mustern der Mobilisierung, politischen Konflikten, Imaginationen, Symbolen und sozialen Signifikanten ansetzen, die in den politischen Kulturen etabliert sind. Die Erscheinungsformen der neuen Protestbewegungen lassen sich aus verschiedenen sozial- und kulturwissenschaftlichen Perspektiven untersuchen, die für sich genommen wertvolle Aspekte beleuchten:

- Die politische Soziologie und Bewegungsforschung thematisiert die Sozialstruktur der Bewegungen und untersucht, wie sich die soziale Träger"innenschaft der Bewegungen zusammensetzt und welche sozialen, aber auch politisch-ideologischen Milieus in ihnen engagiert sind.

- Politik- und sozialwissenschaftliche Analysen untersuchen die politische Funktionalität dieser Bewegungen im Rahmen einer Krise der politischen Repräsentation, in der sie ein tiefes Misstrauen artikulieren und eine neue politische Kultur hervorbringen, die auf die Mobilisierung von Angst und Affekten abzielt. Sie versuchen Zorn, Misstrauen und Ressentiments, kurzum die „politische Wirkmächtigkeit negativer Gefühle“ (Koppetsch 2018; Mishra 2019), für ihre Zwecke zu instrumentalisieren. Jene Affektpolitiken werden auch im Kontext des Auflebens neuer Form von Autoritarismus erörtert (Nachtwey/Frei/Schäfer 2020).

- Andere Studien untersuchen den Wandel auf der Ebene der politischen Ideologien und versuchen einen neuen rechten Denkstil zu bestimmen, der unter Zurückweisung der vermeintlich hegemonialen Ideologien des Kulturmarxismus, der Gender-Ideologie und der political correctness eine "konservative Rebellion“ im Namen individueller Freiheitsrechte anstrebt (Nachtwey/Heumann 2019). Zudem lassen sich neuartige politische Affektkulturen beobachten, die teilweise schon vor dem Aufkommen der Protestbewegungen gegen die Covid-19 Maßnahmen zu beobachten waren und die sich nun prägnant und in bunten Farben zeigen. Die beliebten Selbstbilder der Rebellin und des heroischen Querdenkers bringen zum Ausdruck, was die Text- und Sinnproduktion, die Slogans und Narrative im Umfeld der Mobilisierungen suggerieren: ein affektiv aufgeladenes Weltbild, in dem ein kleiner erlesener Kreis von Nicht-Blinden die Machenschaften der unterdrückenden Elite durch- 
blickt und in der Haltung der radikalen Kritik rebelliert (Gess/Amlinger 2021).

- Sozial- und individualpsychologische Perspektiven setzen sich mit den psychodynamischen Aspekten jener Affektkulturen und ihrer identitätsstiftenden Funktion auseinander. Sie beleuchten den Zusammenhang zwischen kulturell-kognitivem Programm, politischen Affekten sowie der entlastenden Funktion der manichäischen Weltbilder. Sie analysieren, wie die welterklärenden Narrative, denen Verschwörungsmythen zugrunde liegen, ressentimentgeladene Blicke konstruieren, die obsessiv auf (vermeintlich) repräsentative Begebenheiten der politischen Realität fixiert sind (z. B. Sündenböcke), alternative Weltordnungen herbeisehnen oder auch den Untergang der gegenwärtigen Welt heraufbeschwören (Lamberty/Rees 2021).

- Die Affektdispositionen und ihre Verbindung zu narrativen Strukturen werden in kulturwissenschaftlichen Perspektiven beleuchtet, die sich mit dem medialen Kontext auseinandersetzen und dem Einfluss der Narrativik und Ästhetik der Gegenwartskultur nachspüren (Butter 2018, Blume 2020). Sie untersuchen die Interaktion zwischen der sozialen Fantasie, den Imaginationen der Gegenwart und fiktionalen Genres ${ }^{1}$, wobei jener Nexus ausgehend vom Zusammenhang von Medialität und Identifikation beleuchtet wird.

- Aus kommunikations- und medienwissenschaftlicher Perspektive werden die neuen Potenziale der digitalen Kommunikationstechnologien sowie ihre Auswirkungen auf die Herausbildung und Erhärtung von Mustern der Wahrnehmung gesellschaftlicher Realität und politischer Identifikation untersucht. Der (manipulative) Umgang mit „Wissen“, die Verstärkung und Erhärtung von Interpretationsmustern (wenn auch fraglichen Realitätsgehaltes) in kommunikativen bubbles wie etwa Telegram-Gruppen kann den rigiden Glauben an „alternative Fakten“ und die Ambiguitätsintoleranz verstärken. Die neuen sozialen Medien stellen bedeutende Multiplikatoren für die missionarischen Botschaften radikaler communities dar, was diesen Botschaften größere Reichweiten und die Rekrutierung neuer Mitglieder ermöglicht.

1 Die Frage, inwiefern die Fiktionalisierungen Subjektformen suggerieren, die in der außerfiktionalen Welt eine soziale Energie entfalten und dort Modellcharakter erhalten, ist hier zentral und wäre an der Grenze zwischen künstlerischer Fiktion, Populärkultur und den Symbolen der politischen Kultur auszuloten. An dieser Stelle soll nur der Tatbestand erwähnt werden, dass Filme wie „Matrix“ sowie „V wie Vendetta" sich in verschwörungstheoretischen Kreisen einer großen Beliebtheit erfreuen. Generell hierzu: Butter (2018) sowie Boltanski (2015). 
Die erwähnten Facetten verdeutlichen die Vielfältigkeit des Untersuchungsgegenstands der neuen Protestkulturen, wie auch der auf ihn bezogenen Forschungsperspektiven. Im Folgenden werden anhand einer kursorischen Rekonstruktion von Beispielen aus Argentinien und Brasilien die Heterogenität dieser Bewegungen, wie auch ihnen gemeinsame Züge veranschaulicht. Der Schwerpunkt liegt dabei auf den Narrativen, Symbolen und Signifikanten der Bewegungen, anhand derer sie ihre Realität konstruieren und die formativ für ihre Protest- und Affektkultur sind. Von besonderem Interesse ist die Frage, inwiefern sich in diesen Bewegungen eine neue Spielart der politischen Rechten manifestiert, die möglicherweise eine Modernisierung und Aktualisierung der klassischen Rechten Lateinamerikas anzustoßen vermag.

\section{Phänomenologie der neuen Rechten in Lateinamerika}

Wie es „die Rechte“ Lateinamerikas nicht gibt, kann es ebenso wenig „die neue Rechte“ geben. Die politische Rechte Lateinamerikas ist in sich heterogen und trägt, je nach Ausdifferenzierung des nationalen politischen Koordinatensystems, unterschiedliche Züge, umfasst verschiedene Ideologien divergierender Ausrichtung und Radikalität.

Mit Blick auf das deutschsprachige politische Vokabular, das zur Auszeichnung von politischen Tendenzen der Rechten in Europa verwendet wird, sind zunächst sprach- und kulturbedingte Beschränkungen zur Kenntnis zu nehmen, denn die zur Kennzeichnung der Rechten verwendeten Schlagworte wie Nationalismus oder Populismus sind in Lateinamerika mit einer grundlegend anders konnotierten Semantik ausgestattet ${ }^{2}$.

2 Beide Schlagworte, nacionalismo wie populismo, tragen in der lateinamerikanischen Politik andere Konnotationen als in Europa. Während in Lateinamerika Populismus aus liberaler und konservativer Warte verwendet wird, um den Regierungsstil progressiver Linksregierungen (und deren Korruption etc.) zu kritisieren, auf der anderen Seite aber teilweise auch als Selbstbild für eine Politik fungiert, die auf das „Populare“ und die „Volksklassen“ Bezug nimmt, funktioniert „Populismus“ in Europa überwiegend als pejorative Kategorie, mit der (zuweilen in totalitarismustheoretischer Manier) eine Bedrohung des demokratischen Systems bezeichnet wird. In dieser Begriffsfassung stellen Linkspopulismus (der in Europa ja ohnehin eher rudimentär ist, z. B. im Fall der spanischen Partei Podemos) und Rechtspopulismus (AfD, Victor Orban etc.) familienähnliche Phänomene dar, deren gemeinsamer politischer Kern in der Gegnerschaft gegen das etablierte demokratische System besteht. Die unterschiedlich gelagerte Terminologie und die darin sich manifestierenden differenten politischen Kulturen wären bei nationen- und 
Eine Ausdifferenzierung der Schattierungen der Rechten in den politischen Landschaften Lateinamerikas, deren Entwicklung Zyklen „beständiger Pendelausschläge“ (Boris 2020) zwischen Links- und Rechtstendenzen durchläuft, wird im Folgenden nicht vorgenommen. Ebenso wenig soll eine systematische Verortung der Bewegungen im Feld der lateinamerikanischen Rechten geleistet werden (hierzu: Bringel et al. 2020 sowie Eser/ Witthaus 2020) oder wird gar der Anspruch erhoben, den geläufigen Kategorien des reaktionären Autoritarismus, autoritären Neoliberalismus und liberalen Konservatismus einen weiteren Typus an die Seite zu stellen. Es ist vielmehr der Anspruch, die Verwendung konspirationistischer Narrative und ihr Widerhall in neurechten politischen Kulturen in Lateinamerika auszuloten.

\section{Neurechte Verschwörungsmythen in Lateinamerika}

\subsection{Brasilien}

Brasilien ist auf der internationalen Landkarte der extremen Rechten seit dem Wahlsieg Jair Bolsonaros ein stabiler wie bunter Fleck. Ohne auf die Gründe des Wahlsiegs, auf das politische Programm und die Allianzen genauer einzugehen (Boris 2018; Prutsch 2019), kann festgehalten werden, dass sich Bolsonaro im Umgang mit der Corona-Pandemie vor allem dadurch hervortat, dass er die Gefahr der Pandemie offensiv infrage stellte und angesichts der raschen Verbreitung des Virus in Brasilien ein verantwortungsloses Krisenmanagement betrieb. Die Signale, die von seiner Regierung ausgingen, bestanden zunächst in der Indifferenz gegenüber dem Leiden und Sterben der besonders vulnerablen Teile der Bevölkerung und später, im Laufe der Pandemie, die schleichende Gewöhnung an die hohen Todeszahlen.

In seiner Kommunikation imitiert Bolsonaro den Stil des ehemaligen US-amerikanischen Präsidenten Donald Trump, der in einer Mischung aus Verleugnung der Pandemie und offensiver Infragestellung seriöser wissenschaftlichen Forschung und der Ratschläge der gesundheitspolitischen Berufsverbände eine Kommunikationsstrategie der Desinformation qua „alternativer Fakten“ prägte. Wie sein Vorbild zitiert Bolsonaro Verschwö-

kontinentübergreifenden Analysen rechter Tendenzen zu berücksichtigen. Zum Versuch vergleichende Blicke auf die Rechtstendenzen Lateinamerikas aus einer europazentrierten Perspektive zu werfen: Wiegel (2020). 
rungsfantasien an und stellt unbegründete Vermutungen über obskure Zusammenhänge an, die hinter der Pandemie stünden. So bestritt er von Beginn an die Gefahr des Virus, bei dem es sich bloß um eine schwache Form der Grippe, eine gripezinha, handele. Zugleich hat er das Virus als „Fantasie" bezeichnet und als Vorstellungsresultat der Hysterie dargestellt. Er hat sich als Maskengegner positioniert und geweigert, in der Öffentlichkeit eine Maske zu tragen. Entgegen dem Rat seiner Ärzte und der Anordnung lokaler Sicherheitsbehörden nahm er an Großveranstaltungen seiner Anhänger*innen teil. Die in vielen Ländern konsequent umgesetzten Maßnahmen zur Eindämmung der Pandemie lehnte er schon früh als „Extremismus“ ab (GZH 2020) und schlug als Maßnahme gegen das Virus die Verwendung des für diesen Zweck nicht getesteten Malariamedikaments Chloroquin/Hydroxychloroquin vor. Er erklärte öffentlich, sich nicht impfen zu lassen, da Impfungen das Schlimmste seien, was dem Immunsystem des Menschen passieren könnte. Zudem verbreitete er die Verschwörungsvorstellung, das Virus sei von fremden Staaten freigesetzt worden, um die Weltwirtschaft zu schwächen, sowie um ihm und Donald Trump Schaden zuzufügen (La vanguardia vom 12.10.2020). Bei den zitierten Einschätzungen Bolsonaros lassen sich Muster wiedererkennen, die aus der hiesigen Szene der Querdenker*innen-Bewegung bekannt sind. Sie lassen sich nicht bloß auf den exzentrischen Charakter der Person und seinen eigenwilligen Kommunikationsstil zurückführen.Vielmehr ist zu beobachten, dass das ideologische und institutionelle Umfeld des Blocks des Bolsonarismus diese Visionen und Einschätzungen teilt und sogar noch verstärkt.

Als Guru und geistige Inspirationsquelle Bolsonaros gilt der rechtskonservative Journalist und Essayist Olavo de Carvalho, der ein zentraler Vordenker der brasilianischen neuen Rechten ist. Er lebt in den USA und ist ein bedeutendes Verbindungsglied zum Gedankengut der Alt-Right-Strömung und verschwörungsmythologischen Milieus in den USA. Er ist konsequenter Kritiker der „Gender-Ideologie“ und des neurechten Schreckgespenstes des „Kulturmarxismus“, den er im intellektuellen Feld Brasiliens als hegemonial erachtet ${ }^{3}$. Er wirkt in verschwörungstheoretischen Zusam-

3 Der,Kulturmarxismus' beschreibt für die neue Rechte ein Dekadenzsymptom der Gegenwart, demzufolge die Ideologie der Diversity, Multikulturalismus und Globalismus darauf abzielt, die Moral, Familie, Kirche und Nation zu schwächen. Der neurechte Kampfbegriff, der auch von dem rechtsextremen Terrorist Anders Breivik in seinem "Manifest" verwendet wurde, stammt aus den USA, wo er in den 1990er Jahren gegen jene Akademiker*innen in Anschlag gebracht wurde, die vermeintlich durch Sprachpolitik und Denkverbote die Bürger*innen zu besseren 
menhängen, so im rechtsextremen Netzwerk der The John Birch Society und tritt als umtriebiger Journalist und Meinungsmacher in Erscheinung. Seiner Meinung nach ist die Erzählung des Klimawandels die Geburt einer marxistischen Verschwörung. Brasiliens Außenminister Ernesto Araújo ist eingefleischter Bolsonarist. Er vertritt antiprogressistische Positionen und kommuniziert Verschwörungsandeutungen. So attackiert er die Weltgesundheitsorganisation (WHO) und behauptet, dass es sich bei der CoronaPandemie um eine kommunistische Verschwörung zur Schwächung des Kapitalismus handle. Auch die akademische Wissensproduktion attackiert Araújo. Nicht mehr die Universitäten, Wissenschaftler*innen, Intellektuellen, Künstler*innen und Medien sollten die gesellschaftlichen Diskurse gestalten, sondern der vermeintlich gesunde Volksverstand der Bolsonaristen.

Die Intellektuellen- und Wissenschaftsfeindlichkeit harmoniert mit der (Pseudo-)Religiosität des Bolsonarismus, die sich auch in seinen kulturellen Formen sowie in den Bündnissen mit den evangelikalen Kirchen wiederfindet. Die pseudoreligiöse Komponente kommt in der fanatischen Verehrung Bolsonaros durch seine Anhänger*innen zum Ausdruck wie auch in seinem Selbstbild. Im Präsidentschaftswahlkampf hat er sich als Retter der Nation präsentiert, der Brasilien gegen den Kommunismus, den PT (Partido dos Trabalhadores), den ,Kulturmarxismus' etc. verteidigt. Schon vor der Wahl wurde er als „Mythos“ bezeichnet. Medienkampagnen evangelikaler Kirchen inszenierten ihn als Retterfigur, der das Land angesichts der Bedrohung durch Lula, dem Symbol des Satans und der kommenden Apokalypse, schützen werde ${ }^{4}$. Zudem schreiben seine Anhänger“innen dem "rechtspopulistischen Messias“ (Prutsch 2019: 130) zu, gegen das Establishment zu kämpfen und nicht davor zurückzuschrecken, unbequeme Wahrheiten auszusprechen. Die Allianz mit den Pfingstkirchen ist eine Besonderheit des neurechten politischen Projekts des Bolsonarismus. Sie ist zwar auch in anderen lateinamerikanischen Ländern und in den USA zu beobachten, im Falle von Bolsonaro findet sie jedoch eine besonders dichte Ausprägung. Die Verstrickung mit Strömungen dieses religiösen Milieus ist breit und fast schon organisch. Bolsonaro verbündete

Menschen erziehen wollen (Hoppe, Nico: „Kulturmarxismus: Warum der neue Begriff ein großes Missverständnis ist“, in: NZZ vom 26.10.2020).

4 Prutsch zufolge haben evangelikale Kirchen ungefähr 50 Millionen Brasilianer*innen dazu aufgerufen, Bolsonaro zu wählen, um die Wiederkunft des Satan durch Lula zu verhindern; offensichtlich war der Wahlaufruf mit der Fake News verbunden, dass Lula 50 Stiere hat opfern lassen, um Satan ihm wohlgesonnen zu stimmen (Prutsch 2019: 133). 
sich vor seiner Wahl mit Edir Macedo, dem selbsternannten Bischof der Igresia Universal do Reino de Deus und milliardenschweren, sowie einflussreichen Medienunternehmer, dem zahlreiche Fernsehkanäle und Radiostationen gehören. Bedeutende Repräsentant*innen der Pfingstkirchen sind hohe Funktionäre des Bolsonarismus. So ist Silas Malafaia, einer der einflussreichsten evangelikalen Priester in Brasilien, ein Vertrauter Bolsonaros und die Ministerin für Frauen, Familie und Menschenrechte ist die rechtskonservative Predigerin Damares Alves. Fußend auf dieser organischen Verbindung hat Bolsonaro direkt nach der Wahl bei seiner ersten öffentlichen Rede seinen Regierungsauftrag unter das Mandat Gottes gestellt und bestätigte seinen Wahlkampfslogan, demzufolge Brasilien über allem und Gott über allen stehen solle. Im Amt hat er weiter an seinem MessiasImage gearbeitet (Oualalou 2019).

Die religiösen wie verschwörungsmythologischen Narrative liefern dem Bolsonarismus ideologische Pfeiler, die im gegenwärtigen Krisenkontext verstärkt auf Anklang stoßen. Die Skepsis gegenüber der Wissenschaft und die heftige Kritik an den von offiziellen Ärzte- und Gesundheitsverbänden befürworteten Maßnahmen stoßen nicht nur in den evangelikalen Milieus auf Zustimmung. Die dort tief verankerte Impfskepsis ist gleichwohl im Vergleich zur Gesamtbevölkerung, aber auch zu anderen Glaubensgemeinschaften weit überdurchschnittlich (Eisele 2021). Die Zurückweisung der Maßnahmen sowie der Impfung ist vor dem Hintergrund des in den evangelikalen Kirchen propagierten Hyperindividualismus zu verstehen, für den die Freiheit und Verantwortung des Einzelnen im Zentrum steht. Hinzu kommt eine Bibelinterpretation, die starkes Vertrauen in den Beistand Jesus' vermittelt, auch im Fall einer Infektion ${ }^{5}$. Die wissenschaftsfeindliche Mentalität, die sich im aktuellen Covid-Krisenkontext verstärkt ausdrückt, macht die religiösen Milieus empfänglicher für zirkulierende Verschwörungsmythen. Die Sinnangebote der religiösen wie verschwörungsmythologischen Narrative, die untereinander strukturelle Analogien aufweisen, finden im Krisenkontext verstärkten Anklang 6 . Sie bieten einfache Erklärungsmuster an, deren Wirkung aus psychologischer Perspektive

5 Bolsonaros provokant vorgetragene Verweigerungshaltung gegenüber medizinisch-epidemologischen Erkenntnissen trifft sich hier mit der generellen Skepsis, die in evangelikalen Kreisen gegenüber wissenschaftlichen Erkenntnissen (z.B. die Zurückweisung der Evolutionstheorie) vorherrscht, wenn der biblische Schöpfungsbericht den wissenschaftlichen Erkenntnissen als alternatives Narrativ gegenübergestellt wird.

6 Verschiedene Studien haben gezeigt, dass der religiöse Glauben im Krisenkontext zugenommen hat (Hillenbrand 2021). 
als effizienter Abwehrmechanismus beschrieben werden kann. Die angebotenen Sinndeutungen helfen dabei, die eigene Verunsicherung zu verarbeiten und möglicherweise erlittene schmerzhafte Verluste oder durch das Pandemiegeschehen entstandene Schwierigkeiten im Alltag subjektiv zu bewältigen (Eisele 2021). Der mit der Bewältigung der Komplexität des Außen überforderte psychische Apparat der Einzelnen, die sich dem Weltund Pandemiegeschehen hilflos ausgeliefert fühlen, ergibt eine Situation, in der die religiösen Narrative und Verschwörungserzählungen mit ihren Erklärungsmustern (Verschwörung böser Mächte, Virus als Erfindung), Feindbildern (Bill Gates, WHO) und auch einfachen Lösungsangeboten (Trump, Bolsonaro) sowie Heilsperspektiven (Jesus, Messias) attraktive Sinnangebote mit narrativer Evidenz anbieten. Die Verschwörungsszenarien locken damit, über eindeutige Ursachen aufzuklären. Sie deuten Komplotte an, nennen mögliche Schuldige und Verantwortliche, die das Böse repräsentieren. Diese Imaginationen weisen zuweilen dramatische Töne auf, entwerfen apokalyptische Situationen und kommunizieren Paranoia. Sie rufen Empörung hervor und suggerieren die Notwendigkeit von Entscheidungen in der Gegenwart. Inmitten der Ohnmacht blitzen Momente einer Handlungsmacht auf, für die der Coronarebell Bolsonaro das „Modell" liefert.

Die Verbreitung verschwörungsmythischer Narrative in der brasilianischen Bevölkerung erfolgt im Zusammenhang mit der Streuung von FakeNews und Verschwörungsandeutungen von regierungsoffizieller Seite. Die Kommunikationswege der spirituell-religiösen Netze der evangelikalen Kirchen dienen mitunter auch zu ihrer Verbreitung und Sedimentierung. Dem Widerhall dieser Narrative sind kulturelle Dispositionen und Glaubenssysteme zuträglich, die in den Alltagskulturen, in der Volksreligiosität, sowie in anderen Formen von Spiritualität und magischem Glauben in den popularen Sektoren Brasiliens eine gewisse Präsenz haben ${ }^{7}$.

7 Wie in anderen lateinamerikanischen Ländern, sind im kollektiven Unbewussten in Brasilien Formen des magischen Denkens auch durch literarische und kulturelle Traditionen fest verankert. Als ein Beispiel hierfür kann der in Brasilien weit verbreitete Sebastianismus gelten, der sich um den Glauben an die magische Wiederkehr des Ende des 16. Jahrhunderts verschwundenen portugiesischen Königs Sebastian dreht. 


\subsection{Argentinien}

In Argentinien trägt der neurechte, konspirationistische Bewegungstypus andere Facetten. Die Situation ist auch deshalb anders gelagert, da sich die entsprechende Bewegung in der Opposition befindet und ihre Stärke und Ratio aus der Mobilisierung gegen die Pandemiebekämpfungspolitik der (links-)peronistischen Regierung von Alberto Fernández erlangt. Folglich weist die Bewegung andere Schattierungen auf. Ihre Allianzpartner kommen teilweise aus dem Feld der konservativ-liberalen Opposition im Umfeld des Wahlbündnisses Cambiemos um den 2019 abgewählten Präsidenten Mauricio Macri. Aus diesem Spektrum haben Einzelpersonen versucht, Unmutsregungen im Volk für ihre Zwecke zu instrumentalisieren, allerdings kann nicht von einer organischen Verbindung mit der Bewegung die Rede sein.

Am April 2020 setzte ein Protestzyklus gegen die Regierung ein, die zu dieser Zeit noch kein halbes Jahr im Amt war und gerade die Verlängerung der Regelung der Kontaktbeschränkung beschlossen hatte. Der Protest richtete sich gegen den „Autoritarismus“ der Regierung, Anfang Mai zogen wiederum Tausende an die zentrale Plaza del Mayo. Argentinische Nationalfahnen prägten das Erscheinungsbild. Forderungen nach der Möglichkeit, die Geschäfte wieder zu öffnen, waren neben Plakaten zu sehen, die die Pandemie als infame Lüge denunzierten. Bill Gates und Georg Soros wurden wie die „Neue Weltordnung“ zum Gegenstand der Kritik. Ein beliebter Slogan lautete: „Wir wollen keinen Kommunismus”. Diese ersten Proteste waren der Startpunkt breiter und landesweiter Bewegungen. Allein in Buenos Aires fanden zwischen Mai 2020 und Mai 2021 knapp 20 Demonstrationen statt.

Zentraler Gegenstand der Kritik war das Krisenmanagement der Regierung. Anders als in Brasilien befand sich die konspirationistische neue Rechte in Argentinien von Anfang an in der Opposition und verschränkte sich mit den verschiedenen Kritikmustern an den biopolitischen Maßnahmen der Fernández-Regierung. Die Bewegungen zeichnen sich durch eine große soziale wie politisch-ideologische Heterogenität aus: konservative, (neo-)liberale Rechte finden sich hier neben Fraktionen der extremen Rechten, spirituellen Kreisen der Esoterik, der alternativen Medizin und der Anthroposophie. Die politische Opposition aus dem Umfeld der Vorgängerregierung beteiligte sich an Desinformationskampagnen, ging dabei jedoch kaum über das Maß hinaus, das die polarisierte politische Öffentlichkeit Argentiniens seit Jahren kennzeichnet. Der ehemalige Regierungschef Macri äußerte sich im März 2020 auf einem Treffen der liberalen und konservativen Rechten in Guatemala, kurz nachdem die WHO den 
Ausbruch einer globalen Pandemie deklariert hat, dass der „Populismus gefährlicher sei als das Coronavirus“ (Página 12 vom 04.03.2020). Elisa Carrió, eine altgediente Politikerin der liberalen Rechten, die stets polarisierend in die öffentlichen Debatten interveniert, skandalisierte die Entscheidung der Regierung Fernández im November 2020, für die argentinische Impfkampagne den russischen Impfstoff Sputnik-V anzuschaffen und suggerierte ein politisches Bündnis mit Venezuela, Kuba, Ecuador und anderen „pseudosozialistische Halbdemokratien“, das die Vorherrschaft Russlands über Lateinamerika etablieren sollte (ámbito vom 04.06.2021).

Mit der Entscheidung für den russischen Impfstoff erlebten altbekannte, antikommunistische Zerr- und Schreckensbilder eine Renaissance. Die Imagination, dass Argentinien in ein autoritär regiertes, kommunistisches Land transformiert, zu einem zweiten Venezuela werde, wurde zu einem beliebten Topos der Opposition. „Ich akzeptiere keine KommunismusÜbertragung" wurde zu einem populären Slogan, der eine generelle Skepsis gegenüber der Impfung mit weltanschaulich-ideologischer Ablehnung gegenüber diesem spezifischen Impfstoff verbindet (Medina 2020).

Die Kommunikationsstrategie eines Großteils der Opposition besteht darin, Zweifel und Misstrauen gegenüber der Regierungspolitik zu säen und deren Handlungen zu skandalisieren. Manche Kritiken spielen hingegen auf große politische Zusammenhänge an, die einen Verrat des Landes und eine Schädigung, gar eine „Vergiftung“" ${ }^{8}$ der eigenen Bevölkerung suggerierten. Neben der polemischen Rhetorik der Opposition auf dem Feld der argentinischen Politik, auf dem die politischen Auseinandersetzungen klassischerweise mit harten Bandagen ausgetragen werden, regt sich das neurechte Milieu des Konspirationismus vornehmlich auf der Straße und in den sozialen Medien. Es kann partiell an der "offiziellen“ Oppositionsrhetorik, vor allem an den antikommunistischen Schreckensfantasien, wie auch an etablierten Kritikformeln („Autoritarismus“, „Vergiftung“) anknüpfen. Auch wenn schon die antikommunistische Rhetorik deutliche Züge einer paranoiden Verschwörungsvision trägt, weist der Konspirationismus der neurechten Mobilisierung eine neue Qualität auf. Sein „Komplottbaukasten" (Butter) geht weit über das Herunterspielen der Gefahr des Coronavirus hinaus. Es wird ein Komplott im Zusammenhang mit dem Virus, seinem Auftauchen, sowie der Strategien seiner Bekämpfung

8 Carrió reichte im Dezember 2020 Klage gegen Fernández und weitere Regierungsbeamte ein. Der Vorwurf war, dass mit dem Sputnik-Impfstoff eine Vergiftung („envenenamiento“) der argentinischen Bevölkerung betrieben werde (Calloni 2020). Die Auswahl von Sputnik V sei zudem ein großer nationaler Betrug („gran estafa nacional“). 
suggeriert. Der Hinweis auf (teils genannte, teils unbestimmt bleibende) externe Faktoren ist ein durchgängiges Motiv. Es tauchen die transnational geläufigen Symbole wie Einzelpersonen auf, denen eine im CovidPandemiekontext zweifelhafte Rolle zugeschrieben wird (Gates, Soros). Die entsprechenden Feindbilder, die deutliche Verschwörungsanspielung kommunizieren, werden mit konkreten Bezugnahmen auf die argentinische Politik adaptiert: „Ich habe Alberto und nicht Soros gewählt“ lautet einer der zahlreichen Slogans. Ähnliches ist der Fall bei Neologismen wie plandemia (Plan-demie) oder der infektadura (das „k" fungiert im politischen Vokabular Argentiniens als Bezeichnung für den Kirchnerismus) sowie dem impfkritischen Slogan „Die Impfung ist Gift“. Diese kurzen Slogans und Wortschöpfungen verbreiten Komplottanspielungen und suggerieren einen auf die Pandemie bezogenen Plan, der auf die Schädigung der Bevölkerung abzielt (drastisch ausgedrückt in der Giftmetaphorik).

Bei den Protesten in Argentinien ist eine Gruppierung besonders aufgefallen, die so genannten Medicos por la Verdad („Ärzte für die Wahrheit"). Sie haben eine große mediale Sichtbarkeit erlangt und werden im Folgenden als symptomatischer Fall betrachtet. Es handelt sich bei den medicos um Fachkräfte des Gesundheitswesens ${ }^{9}$, die sich gegen die Maßnahmen der Pandemiebekämpfung und die Impfung wenden. Wie ihre internationalen Partnerorganisationen ${ }^{10}$ geben sie vor, die Menschen vor bevormundenden Maßnahmen der Pandemiebekämpfung zu schützen und versuchen ihren fundamentalen Zweifeln am Krisenmanagement durch Verweis auf ihre professionelle Ausbildung und Herkunft Gewicht und Plausibilität zu verleihen. Sie inszenieren sich als isolierte Stimmen der Kritik, die unbequeme Wahrheiten aussprechen, die von Politik und Medien willentlich verschwiegen werden. Ein renommiertes Gesicht dieser Organisation ist die Ärztin Chinda Brandolino, die zahlreiche Falschinformationen über das Corona-Virus verbreitet hat. Sie hat auf facebook über 133.000 Follower*innen (Stand Juli 2021) und agitiert als Rednerin auf

9 Die Gruppierung ist relativ klein, hat aber eine starke mediale Präsenz, so dass die von ihnen in Zirkulation gebrachten Krisennarrative breit wahrgenommen werden. Mehr als 55 professionelle Beschäftigte im Gesundheitssektor - Ärzt*innen, Biochemiker*innen, Psycholog*innen und Ernährungswissenschaftler"innen - sind zuletzt öffentlich aufgetreten, um die Maßnahmen in Frage zu stellen und alternative Therapien vorzuschlagen (Micheletto 2021a, 2021b).

10 Die argentinische Gruppierung ist Ableger des gleichnamigen spanischen Kollektivs, das wiederum die homologe Organisation der deutschen Ärzte für Aufklärung (ÄfA) ist. In Argentinien sind zudem noch die Epidemólogos Argentinos Metadisciplinarios in Erscheinung getreten, Stoßrichtung und Stil ihres Engagements weisen ähnliche Züge auf. 
Demonstrationen wie auf Online-Vorträgen. Schon vor der Corona-Pandemie war sie öffentlich als scharfe Kritikerin des Schwangerschaftsabbruchs aufgetreten. In ihr überkreuzen sich ultrakonservativer Katholizismus, die radikale Kritik an den Maßnahmen sowie die Verwendung von Verschwörungsmythen ${ }^{11}$. Ihre Auftritte enthalten gezielte Provokationen. Sie verwendet eindeutig konnotierte und historisch-affektiv aufgeladene Schlagwörter, deutet sie um, um ihre Kritik in dramatisierende Darstellungen $\mathrm{zu}$ kleiden und alternative Sichtweisen auf das Pandemiegeschehen zu konstruieren, die das Vertrauen in die Politik unterminieren. Die Absicht, durch verbale Offensiven die Realität in ihren Grundfesten in Frage zu stellen und durch krude historische Vergleiche Konfusion zu stiften, ist ein Zug, der auch in anderen Kontexten der konspirationistischen neuen Rechten zu beobachten ist ${ }^{12}$.

Dem Namen der Organisation und dem darin ausgedrückten Wahrheitspathos entsprechend, inszenieren sich die médicos als „Rufer in der Wüste“, die mit ihren eigenen Wahrheiten die Lügen der Regierung(en), ihre Verstrickungen und Agenden aufdecken. Der Augenarzt (!) Mariano Arriaga ist ein weiteres Gesicht der médicos, der dieses Pathos inszeniert. Er führte einen „Ritt für die Wahrheit“ durch das argentinische Hinterland durch mit dem Ziel seine impfkritische Nachricht und "die Wahrheit“ ins Land hineinzutragen. Er zog von Dorf zu Dorf und hielt auf zentralen Plätzen Reden ab. Arriaga gibt sich ein ländliches Outfit und zitiert im Rahmen seiner öffentlichen Auftritte auf kauzische Art mystische und nationalistische Texte (Micheletto 2021a). Zu seinem exzentrischen Auftreten tritt seine wechselhafte Biografie, die von Kunstfehlern, Verfahren ge-

11 Auf virtuellen Treffen der antivacunas wettert sie gegen die Impfkampagne, die zwangsweise auf die argentinische Bevölkerung angewandt werde oder stellt die Pandemie als eine geplant eingesetzte Biowaffe dar (Ziegler 2020). Die Verwendung der Mund-Nasen-Maske lehnt sie rigide ab, da deren Verwendung gravierende gesundheitliche Konsequenzen für das Immunsystem habe. Ein in ihrem Namen unterzeichnetes Flugblatt ruft dazu auf, im öffentlichen Raum nicht die Maske zu tragen, um sich nicht zu Kompliz*innen der als Wissenschaft verkleideten Diktatur zu machen („No seas cómplice de esta DICTADURA VESTIDA de CIENCIA“). Diese Verweigerungshaltung sei die schnellste und harmloseste Aktion gegen das einzige Virus, das heutzutage zirkuliere: der "Staatsterrorismus“. Mit diesem Terminus („terrorismo del estado“) stellt sich eine polemische Verbindung zu den brutalen Repressionspraktiken der letzten Militärdiktatur in Argentinien (1976-1983) her.

12 Vgl. den Fall der "Jana aus Kassel“, eine junge Aktivistin aus der Querdenker*innen-Szene, die sich bei einer Rede in ihrer Aktivistinnenrolle selbstviktimisierend als Sophie Scholl dargestellt hat. 
gen ihn als Mediziner, Berufseinschränkungen und zahlreichen Niederlassungswechseln gekennzeichnet ist. Sowohl ob die Exzentrik seines Auftretens als auch seine wechselhafte Lebensgeschichte erinnern an die Lebensläufe der männlichen Protagonisten aus der deutschen Verschwörungsszenerie, bei denen ein enger Zusammenhang zwischen narzisstischer Persönlichkeitsstruktur, gekränkter Männlichkeit und Verschwörungsmentalität vermutet werden kann. Das radikale Auftreten des „furchtlosen Helden und Messias" gibt vor, die Wahrheit über die offiziellen Diskurse und Todeszahlen zu entschleiern ${ }^{13}$. Seine heroisch-messianische Performance drückt eine politische Affektkultur aus, in der eine (vermeintlich) radikalkritische Haltung mit einem rebellischen Gestus vermengt wird.

Die médicos verbreiten ihrem Selbstbild entsprechend ,alternative Sichtweisen" auf die Epidemie, die als Desinformationskampagnen bezeichnet werden können. Sie dramatisieren die Risiken der Maßnahmen und Impfungen, verbreiten Falschinformationen und empfehlen die Verabreichung von Hydroxychloroquin. Anknüpfend an das Programm der alternativen Medizin werden starke Zweifel an den Einschätzungen der „Schulmedizin" verbreitet. Ihre radikale Kritik fußt auf paranoiden Unterstellungen und Mutmaßungen über die bösen Absichten der Eliten. Ausgewählte Feindbilder (Soros, Gates, WHO) werden zu Sündenböcken stilisiert. Auf die Existenz von Komplotten wird angespielt, deren Zusammensetzung wird jedoch nicht konkretisiert, so dass es Spielraum für die Verschwörungsgläubigen gibt, diese Leerstelle mittels ihrer eigenen Imaginationskraft auszufüllen. Die Suggestion externer umstürzlerischer Kräfte, die im Bündnis mit der nationalen Regierung ein autoritär-kommunistisches Re-

13 Bill Gates habe beschlossen, die Weltbevölkerung um 90 Prozent zu reduzieren und dies durch Impfung und Abtreibung zu erreichen. Die Mischung aus Opferrhetorik und apokalyptischem Szenario resultiert in einem rebellischen Aufruf, „ein glanzvolles Nein zu zeigen, als Geste des Friedens und der Selbstermächtigung und Bekräftigung der eigenen Wahrheit“ (zit. n. ebd.). In den Reden Arriagas treten Charakteristika der verschwörungsmythologischen Rhetorik auf, die einen geplanten, diktatorischen Zugriff auf das Leben durch den Staat skandalisiert, der in den Interessen der Pharmaindustrie, professioneller Medizinverbände und weiterer nicht näher erläuterten „Agent*innen“ durchgeführt werde. Ungerechtfertigte Freiheitsbeschränkungen werden skandalisiert und die Gesundheitspolitik des Staates als "Terrorismus" und "Autoritarismus" gebrandmarkt. Zugleich werden Zerrbilder der globalen Politik entworfen, internationale Verschwörungen und Pakte insinuiert und durch dieses diskursive Bündel das von Paranoia angetriebene Misstrauen an den offiziellen staatlichen Maßnahmen gesät. Verbunden werden die Komplottvorstellungen mit einem sich radikalkritisch gebenden Gestus der Befreiung und Selbstermächtigung. 
gime zu installieren bestrebt sind, erhalten in den verschwörungsmythologischen Narrativen der sogenannten antivacunas blumige Auskleidungen, die auch aus anderen Ländern schon bekannt sind ${ }^{14}$.

Jene verschwörungsmythischen Milieus, für das die médicos ein symptomatisches Phänomen darstellen, weisen zudem deutliche Züge von Antifeminismus und Antisemitismus auf. Der Antifeminismus verbindet die Milieus der Impfgegner*innen mit religiös-ultrakonservativen Kreisen. Die Äußerungen Brandolinos, die in der Kampagne der Abtreibungsgegner*innen prominent in Erscheinung getreten ist, unterstreichen diese ideologische Allianz deutlich ${ }^{15}$. Der Antisemitismus ist eng mit dem Archetyp der Verschwörungserzählung, dem Narrativ der jüdischen Weltverschwörung, verbunden. Sofern auf Komplotte und globale Verschwörungen angespielt wird, ist die Imagination eines global vernetzten, finanziell und politisch mächtigen Judentums, das die Strippen zieht, um die Welt zu beherrschen, nicht weit entfernt ${ }^{16}$. Die Verschränkung der unterschiedlichen Verschwörungsmythen und Antisemitismus zeigt sich in mehr oder weniger versteckter Form im Rahmen der neurechten Corona-Proteste, so in der Imagination des Komplotts einer „Neuen Weltordnung“ oder in der Hassfigur des Georges Soros. In Argentinien haben sich im Umfeld der Corona-Leugner*innen auch ganz explizite Formen des Judenhasses in Form von Karikaturen und Aufklebern manifestiert, auf denen als Jüd"innen kenntlich gemachte Personen mit der Entwicklung und Vermarktung von Impfstoffen in Verbindung gebracht werden, die dazu dienen die Weltbevölkerung zu beherrschen und eine „Neue Weltordnung“ zu installieren. Das Simon Wiesenthal Zentrum in Buenos Aires hat einen Anstieg

14 Auch ist zu beobachten, dass sich Personen aus der Medien-Öffentlichkeit (wie dies auch in Deutschland der Fall ist), prononciert als Corona-Skeptiker*in positioniert haben, so das argentinische Modell und die Fernsehmoderatorin Ivana Nadal, die auf Instagram zwei Millionen Follower*innen zählt, wo sie die Existenz des Virus leugnet sowie Elemente von Verschwörungserzählungen teilt.

15 Brandolino verwendet auf polemische Weise den feministischen Slogan „Mein Körper, meine Entscheidung“, um die individuelle Autonomie der Bürger*innen in der Impfproblematik zu behaupten. Der feministische Slogan, der die Entscheidungssouveränität der schwangeren Frau über ihren Körper bekräftigt, wird hier zur Freiheit der körperlichen Unversehrtheit im Impfkontext uminterpretiert. Der rebellische Gestus jener progressiven Lösung des Feminismus wird provokativ zu einer Widerstandshaltung umgedeutet, die sich gegen den diktatorischen Zugriff des „Impfstaates“ auf die Körper der Individuen wehrt.

16 Mit den Protokollen der Weisen von Zion liegt die Urversion einer Verschwörungserzählung als „offensichtliches Beispiel für eine Verschwörungstheorie“ vor (Boltanski 2015: 268-76, hier S. 269; Butter 2018: 160-169). 
antisemitischer Praktiken in der Region seit Beginn der Covid-19-Krise und die Zunahme von „Hassgruppen“ in digitalen, sozialen Netzwerken festgestellt (Martínez Ferro/Fernández Bravo 2021).

\section{Schluss}

Verschwörungserzählungen haben im Covid-19-Krisenkontext schlagartig eine große Beliebtheit erhalten. Sie sind wirkungsmächtig, weil sie an fest im kulturellen Unbewussten verankerte literarische und diskursive Traditionen, mythologische Muster und Glaubenssysteme anknüpfen. Als kulturelles System umfassen sie Untergangsfantasien, die drastische Bilder einer dekadenten Welt entwerfen, hinter der die Intention böswilliger Akteur*innen, Agent*innen und „Sündenböcke“ stecken. Für die insinuierten Komplottstrukturen liefern die Verschwörungserzählungen ritualhaft weitere Belege, die in Texten, Handlungen und Symbolen der Gegenwart aufgespürt werden. Ebenso liefern sie fortlaufend Verantwortliche für die Fehlentwicklungen. Sie schaffen „Erzählgemeinschaften“ und Gruppenidentitäten, die sich in der gemeinsamen Agitation, auf der Straße oder in den sozialen Netzen manifestieren. Der rebellische Widerstandsgestus, der die Demonstrationen und Protestäußerungen beseelt, bringt nicht nur die kognitiv-konzeptuelle Dimension der Verschwörungsmythologie zum Ausdruck, sondern veranschaulicht auch deren Performativität, wenn sie Themen setzen, Begriffe und Schlagwörter prägen und Formen von politischer Affektkultur und kollektiver Identität prägen.

Die lateinamerikanische Verschwörungsnarrativik hat in der Vergangenheit eine breite Spanne an Erscheinungen hervorgebracht (Senkman/Roniger 2019). Die Beispiele aus Argentinien und Brasilien weisen auf neuartige Facetten des Phänomens hin, die sich im Kontext der Corona-Pandemie herausgebildet haben und die als neurechte Protestkulturen beschrieben werden können. Vor dem Hintergrund der jeweiligen politischen Situation in beiden Ländern haben sich unterschiedliche Allianzen herausgebildet. In Brasilien kann der polemische Negationsdiskurs des Präsidenten Bolsonaros in seinem Unterstützer*innen-Milieu trotz der desaströsen Konsequenzen seiner Politik immer noch auf Zustimmung zählen, wenn auch diese zunehmend erodiert. In Argentinien ist das Entstehen eines heterogenen oppositionellen Blocks zu beobachten, innerhalb dessen sich verschiedene Akteure mit unterschiedlichen diskursiven Einsätzen an der Pandemiebekämpfungspolitik der Regierung abarbeiten. Das Ziel, das Vertrauen in das Regierungshandeln zu unterminieren, konnte durch eine Strömung kapitalisiert werden, die oppositionelle Des- 
informationspolitik mit der Verbreitung „alternativen Wissens“ und der Lancierung expliziter Verschwörungserzählungen kombinierte. Auch in Argentinien ist zu beobachten, dass die Skeptiker"innen mit religiös organisierten Milieus eine Allianz eingehen und an Teile der konservativ-christlichen Opposition andocken, die sich zuletzt gegen die Liberalisierung des Schwangerschaftsabbruchs formiert hatte. Die Verbindung zu religiösen Milieus ist nur eine Facette dieser neurechten politischen Kultur. Sie weist allerdings sowohl in Brasilien wie auch in Argentinien spezifische Auffälligkeiten auf, für die sich weitere Beispiele in Lateinamerika finden lassen. ${ }^{17}$

In den Protestbewegungen lässt sich das Wirken einer Verschwörungsmentalität beobachten, die eine spezifische politische Sprache sowie Affektkultur strukturiert. Über digitale Medien international vernetzt, hat sich hier ein neuartiger globaler Typus von „Kritik“ und politischer Kultur herausgebildet, der auf einen ähnlichen Fundus von Narrativen, Rhetorik und Symbolen zurückgreift. Der globale Kontext der Verunsicherung im Zusammenhang mit der Covid-Krise hat wie ein Katalysator auf die Entfaltung dieser politischen Kulturen gewirkt. Dem verschwörungsmythischen Kritiktypus und den mit ihm verbundenen Affektkulturen gelingt es, auf subjektive Befindlichkeiten in der Gegenwart des Krisenkontextes zu reagieren und sie zu formen, ihnen Sinnstiftung wie auch symbolisch-kulturellen Ausdruck zu verleihen.

Der globale Krisenkontext bringt tiefgreifende Irritationen und Verunsicherungen breiter Bevölkerungsschichten mit sich. Er steigert das Kontingenzbewusstsein und die Wahrnehmung der Gegenwart als krisenbehaftet, was die Sorge um die eigene Existenz, ihre Vergänglichkeit und die Labilität der körperlichen Gesundheit verstärkt. Als Reaktion auf diese Situation bieten die imaginationsreichen Verschwörungserzählungen Formen

17 Der emeritierte Erzbischof der Diözese Guadalajara Juan Sandoval Iñiguez hat Verschwörungsmythen zum Covid-Geschehen verbreitetet. Er warnt, dass die Impfungen als „Zeichen des Biestes“ einen gefährlichen Mikrochip enthalten, der den Menschen implantiert werde. Ein kleiner Zirkel einer mächtigen Elite hätte die Ausbreitung des Coronavirus vorangetrieben, um so den Plan zur Ergreifung der Weltherrschaft umzusetzen (Saludconlupa o.J.). Die Allianz der politischen Rechten mit verschiedenen religiösen Milieus ist stets in ihrer Ambivalenz zu sehen. Generell ist der Bedeutungszuwachs evangelikaler Gemeinden lateinamerikaweit als Faktor zu bedenken, der auf die Reformulierung der politischen Rechten Konsequenzen hat; dass in Bolivien die Opposition 2019 gegen Evo Morales vorging, um die „Rückkehr der Bibel“ einzuleiten, ist ein weiterer Sachverhalt, der auf das Bündnis der politischen Rechten mit religiösen Milieus hinweist (Boris 2020: 37f.; 46). 
der Kontingenzbewältigung und formulieren Glaubenssätze, die Orientierungsangebote unterbreiten. Sie erklären das unübersichtliche Geschehen, ordnen es, stiften Bilder und Vorstellungen, mit denen die Krisenhaftigkeit des Weltlaufs „bewältigt“ werden kann. Sie wirken wie Mythen, das heißt als „Erzählungen [...], die durch die Imagination einer paradigmatischen, d.h. bedeutsamen Geschichte die Welt raumzeitlich ordnen und damit Handlungsanweisungen für Individuen wie für Kollektive anbieten“ (Atwood, zit. n. Blume 2020: 27). Die kommunizierte Ahnung eines in der Gegenwart wirkenden Komplotts suggeriert eine übersichtliche Konfliktstruktur, die das Individuum imaginär in einen Konflikt mit dem weltweit agierenden Bösen stellt. Der Gestus radikaler Kritik geht mit dem Pathos der Erleuchtung einher. Er suggeriert einen verführerisch einfachen Weg in die "Wahrheit" und den Ausgang aus dem Zustand der Verblendung. In der Geschichte des Verschwörungsdenkens stellen sie einen Typus der Desinformation paranoiden Stils dar, der spirituelle und obskurantistische Folklore aufgreift, Teile der konservativen Milieus zu radikalisieren vermag sowie eine Nähe zur „faschistischen Lüge“ (Finchelstein 2021a, b) aufweist.

Zugleich enthalten diese Erzählungen insofern einen rationalen Kern, als sie auf zentrale Probleme der Gegenwart hinweisen, sie skandalisieren $^{18}$. Die im Covid-Kontext virulent gewordenen Verschwörungserzählungen docken an solche rationalen Intuitionen der Skepsis an, die vormals eher im Kontext links und progressiv akzentuierter Kritikmuster thematisiert wurden ${ }^{19}$. Mit dem neuen Gestus der Radikalkritik beerbt das nach rechts hin offene Projekt der Verschwörungsmythen jene Kritikmuster und integriert Fragmente aus ihnen zugunsten der eigenen narrativen Evidenz. Dass diese Formen der Daseins- und Krisenbewältigung

18 Dies betrifft etwa die schon seit längerem beobachtete „Krise der politischen Repräsentation“, die Verselbständigung der Sphäre des Politischen sowie die Phänomene der institutionalisierten Korruption. Es umfasst aber auch die kritischen Intuitionen gegenüber der Macht- und Verwertungsinteressen der konzentrierten Pharma- und Gesundheitsindustrie (und generell des globalisierten Kapitalismus) sowie gegenüber der ideologischen Rahmung und Verzerrung der medialen Berichterstattung.

19 Untersuchungen zu den Protestbewegungen in Deutschland haben ergeben, dass sich auch „soziologisch“ in den Bewegungen ein gewisser Rechtsdrift ausdrückt. Zahlreiche Aktivist*innen waren früher in der politischen Linken engagiert, weshalb sich diese Protest- und Subkulturen auch als linke Bewegungen mit Rechtsdrift beschreiben lassen, die zunächst durch eine ideologische Heterogenität ausgezeichnet waren und sich später zunehmend die Rechte etablieren konnte (Nachtwey/Frei/Schäfer 2020). 
eine paranoide Modellierung der Realität implizieren und auf paranoide Art apokalyptische Szenarien entwerfen, in denen komplexe Problemlagen durch simple Akteurs- und Komplottstrukturen, Sündenbockfiguren und Verschwörungsfantasien ausgeleuchtet werden, reduziert keineswegs ihr Potenzial, auf die wahrgenommene Verunsicherung und Kontingenz eine „beruhigende“ Antwort zu liefern.

Hinsichtlich ihrer Verbreitung haben sich die sozialen Medien als Katalysator und Resonanzboden erwiesen, durch die jene Erzählungen ein wachsendes Publikum für ihre Szenarien, Komplottsuggestionen und skandalisierenden „alternativen Fakten“ finden. Die bubbles der digitalen Kommunikation und einschlägigen Telegram-Gruppen wirken als Filterblasen von Information und Echokammern bestimmter Überzeugungen. Die digitalen Medien-Milieus ermöglichen, dass sich Menschen mit ähnlichen Verschwörungsaffinitäten vernetzen, ihren Glauben teilen und wechselseitig bestätigen. Sie sind der zentrale Ort der Verbreitung und kollektiven Verarbeitung der Medienbotschaften. Es bilden sich so Bewegungsmilieus heraus, die Kommunikationspunkte und Multiplikatoren eines geteilten Fundus von Narrativen, Orientierungen und Grundüberzeugungen sind.

Inwiefern sich in Lateinamerika dieser neue Typus einer heterogenen rechten Bewegungskultur etablieren und zu einem stabilen Faktor heranreifen kann, der mehr ist als bloße Kuriosität und modische Erscheinung bizarren Anstrichs, ist weiterhin kritisch zu verfolgen. Dies wird auch davon abhängen, ob und inwiefern sich alternative Formen der Politisierung der gesellschaftlichen Verunsicherung und Ohnmachtserfahrungen jenseits der Narrative eines postmateriellen, konspirationistischen Rebellentums herausbilden, auch im Sinne des für Lateinamerika bisher klassischen Typus der "sozialen Bewegung als Form des Unterschichtenprotests“ (Boris 2007). Auch wird sich zeigen, ob und inwiefern sich neue politische Formationen der Rechten auf dem heterogenen Feld des dynamisierten Unmuts herausbilden und etablieren - jenseits der zuletzt stark beobachteten esoterischen Neigungen. Mit Javier Milei hat bei den argentinischen Parlamentswahlen 2021 ein Kandidat einer „neuen Rechten“ einen Achtungserfolg erzielt $(17 \%$ in seinem Wahldistrikt Ciudad Autónoma de Buenos Aires), der sich im Rahmen der Proteste gegen die Pandemiebekämpfungspolitik im Laufe des Jahres 2020 profiliert hatte. Der liberale Ökonom Milei rekurriert nicht auf Verschwörungsmythen, steht der politischen Philosophie des Libertarianismus und Anarcho-Kapitalismus nahe und verteidigt den Wert der „Freiheit" vehement gegen staatliche Interventionen. Er gibt sich als nach rechts offen und nennt Trump und Bolsonaro als seine Vorbilder. Milei könnte in der sich neu organisierenden Rechten 
im südlichen Lateinamerika ein ergänzendes Phänomen zu der esoterisch und verschwörungsmythologisch ausgerichteten Strömung darstellen beide, dies bleibt zu befürchten, werden nicht allzu schnell von der politischen Landschaft verschwinden.

\section{Literatur}

Boltanski, Luc (2013): Rätsel und Komplotte: Kriminalliteratur, Paranoia, moderne Gesellschaft, Berlin: Suhrkamp.

Blume, Michael (2020): Verschwörungsmythen: Woher sie kommen, was sie anrichten, wie wir ihnen begegnen können, Ostfildern: Patmos.

Boris, Dieter (2020): Rechts- und Linkstendenzen in Lateinamerika. Zur ssozialen Grammatik beständiger Pendelausschläge, in: Eser, Patrick; Witthaus, Jan-Henrik (Hg.): Rechtswende in Lateinamerika : Politische Pendelbewegungen, sozioökonomische Umbrüche und kulturelle Imaginarien in Geschichte und Gegenwart, Wien: Mandelbaum, 32-67.

Boris, Dieter (2007): Soziale Bewegungen als Form des Unterschichtenprotests: Das Beispiel Lateinamerika, in: Forschungsjournal Soziale Bewegungen, 20, 81-87.

Boris, Dieter (2002): Macht und Ohnmacht sozialer Bewegungen in Lateinamerika., in: Leviathan, 30, 113-131.

Bringel, Breno; Chávez, Marxa; Gómez, Javier; et al. (Hg.) (2020): NUEVAS DERECHAS AUTORITARIAS. Conversaciones sobre el ciclo político actual en América Latina, Quito: Fundación Rosa Luxemburgo.

Butter, Michael (2018): Nichts ist, wie es scheint: Über Verschwörungstheorien, Berlin: Suhrkamp.

Calloni, Stella (2020): Demandan al presidente de Argentina por "envenenar" con la vacuna rusa, in: La jornada (Mexiko) vom 23.12.2020.

Eisele, Ines (2021): Evangelikale: Gottes Segen statt Corona-Impfung?, in: Deutsche Welle vom 23.04.2021, [https://p.dw.com/p/3rdmB], letzter Aufruf 02.10.2021.

Eser, Patrick; Witthaus, Jan-Henrik (Hg.) (2020): Rechtswende in Lateinamerika : Politische Pendelbewegungen, sozioökonomische Umbrüche und kulturelle Imaginarien in Geschichte und Gegenwart, Wien: Mandelbaum.

Finchelstein, Federico (2021a): La larga trama del conspiracionismo, in: Clarín vom 05.01.2021.

Finchelstein, Federico (2021b): Breve historia de la mentira fascista, Buenos Aires: Penguin Random House.

Gess, Nicola; Amlinger, Carolin (2021): Schamanen gegen die Macht des Virus. Verschwörungstheoriker*innen als Sozialfiguren der Corona-Pandemie, in: KWI-BLOG vom 15.02.2021, [https://blog.kulturwissenschaften.de/schamanen-g egen-die-macht-des-virus/], letzter Aufruf 02.10.2021. 
GZH (2020): Bolsonaro chama de "extremismo" e "histeria" medidas adotadas diante da pandemia do coronavirus, 15.03.2020; [https://gauchazh.clicrbs.com.br/politica/no ticia/2020/03/bolsonaro-chama-de-extremismo-e-histeria-medidas-adotadas-diant e-da-pandemia-do-coronavirus-ck7ts19yw001h01s2j7jncfeb.html], letzter Aufruf 02.10.2021.

Hillenbrand, Carolin (2021): Es geht um die Existenz. Die Pandemie hat religiöse Werte gestärkt, aber die Kirchen profitieren davon nicht. Woran liegt das?, in: Die Zeit vom 30.07.2021, [https:/www.zeit.de/2021/31/glaube-corona-krise-religi on-rueckbesinnung-kirchenaustritt], letzter Aufruf 02.10.2021.

Lamberty, Pia; Rees, Jonahs H. (2021): Gefährliche Mythen: Verschwörungserzählungen als Bedrohung für die Gesellschaft, in: Zick, Andreas; Küpper, Beate (Hg.): Die geforderte Mitte. Rechtsextreme und demokratiegefährdende Einstellungen in Deutschland 2020/21, Bonn: Dietz Verlag J.H.W. Nachf, 283-299.

Medina, Walter C. (2020): La vacuna ,comunista“, in: Nuevatribuna.es vom 04.11.2020, [https://nuevatribuna.publico.es/articulo/global/argentina-orde na-compra-vacuna-rusia-covid/20201104094038180870.html], letzter Aufruf 02.10.2021.

Martínez Ferro, Sol; Fernández Bravo, Ezequiel (2021): Antisemitismo y odio: una encuesta revela que 3 de cada 10 argentinos relacionan a la comunidad judía con el origen del coronavirus, in: Infobae vom 16.07.2021, [https://www.infobae.com /sociedad/2021/07/16/antisemitismo-y-odio-una-encuesta-revela-que-3-de-cada-1 0-argentinos-relacionan-a-la-comunidad-judia-con-el-origen-del-coronavirus/], letzter Aufruf 02.10.2021.

Micheletto, Karina (2021a): 'Médicos por la verdad'que siempre es de derecha, in: Página 12 vom 31.05.2021.

Micheletto, Karina (2021b): El peligro de los negacionistas de la pandemia. Las denuncias por mala praxis que acumulan los grupos antivacunas, in: Página 12 vom 09.06.2021.

Nachtwey, Oliver; Schäfer, Robert; Frei, Nadine (2020): Politische Soziologie der Corona-proteste, in: SocArXiv. Dezember 2020; [doi:10.31235/osf.io/zyp3f], letzter Aufruf 02.10.2021.

Oualalou, Lamia (2019): Los evangélicos y el hermano Bolsonaro, in: Nueva Sociedad, 280, 68-77.

De La Quintana J. (2020): Liberan en Perú a trabajadores secuestrados por hacer mantenimiento a antenas que, según la población, propagan el covid-19; in: CNN vom 16.06.2020, [https://cnnespanol.cnn.com/2020/06/16/alerta-peru-liber an-a-trabajadores-secuestrados-por-hacer-mantenimiento-a-antenas-que-segun-la -poblacion-propagan-el-covid-19/], letzter Aufruf 28.07.2020.

Prutsch, Ursula (2019): Populismus in den USA und Lateinamerika, Hamburg: VSA.

Saludconlupa (o.J): Juan Sandoval Íñiguez, [https://saludconlupa.com/series/desinf ormantes/juan-sandoval-iniguez/], letzter Aufruf 28.07.2020.

Semán, Pablo (2021): Las vacunas y las creencias argentinas, in: eldiarioAR vom 13.02.2021. 
Senkman, Leonardo; Roniger, Luis (2019): América Latina Tras Bambalinas : Teorías Conspirativas, Usos y Abusos, Pittsburgh: Latin American Research Commons.

Wiegel, Gerd (2020): Rechter Aufstieg in Europa - Vergleichspunkte zu Lateinamerika?, in: Eser, Patrick; Witthaus, Jan-Henrik (Hg.): Rechtswende in Lateinamerika : Politische Pendelbewegungen, sozioökonomische Umbrüche und kulturelle Imaginarien in Geschichte und Gegenwart, Wien: Mandelbaum Verlag, 20-31.

Ziegler, Gloria (2020): Ultraconservadores y negacionistas intentan frenar la ampliación de derechos en Argentina, in: ojopúblico vom 20.12.2020. 